\begin{tabular}{|c|c|c|}
\hline & Benha Veterinary Medical Journal & 1). \\
\hline $\begin{array}{l}\text { Official Journal Issued by } \\
\text { Faculty of } \\
\text { Veterinary Medicine }\end{array}$ & Journal homepage: https://bvmj.journals.ekb.eg/ & $\begin{array}{c}x=8 \\
\text { Since } 1990\end{array}$ \\
\hline
\end{tabular}

\title{
The protective effects of Spirulina against Non-alcoholic fatty liver disease induced by high fat diet in rats
}

Samy A. Hussein, Yakout A. El-Senosi, Mohammed k. Mahfouz, Marwa M. Fawzy*. Department of Biochemistry, Faculty of Veterinary Medicine, Benha University, Egypt.

\begin{tabular}{l} 
ARTICLE INFO \\
\hline Keywords \\
Nonalcoholic fatty liver \\
disease, \\
Spirulina, \\
Oxidative stress, \\
Inflammatory mediators, \\
Histopathology. \\
\hline Received $07 / 03 / 2021$ \\
Accepted 26/03/2021 \\
Available On-Line \\
01/07/2021
\end{tabular}

\begin{abstract}
The ultimate goal of this study is to investigate the bimolecular changes in a rat model of high fat diet induced Non-alcoholic fatty liver disease (NAFLD). Also, the lipotropic, antioxidant and anti-inflammatory effects of spirulina platensis treatment were clarified through the evaluation of certain biochemical and molecular parameters in the blood and liver tissue of rats. Twenty one adult white albino rats were separated into three groups. Group I (Normal control): received no drugs and balanced diet, Group II (NAFLD- induced group): rats fed high fat diet for 8 weeks followed by normal basal ration feeding for another 8 weeks. Group III (NAFLD + spirulina): rats treated with spirulina orally at a dose of (150 $\mathrm{mg} / \mathrm{kg}$ b.wt/day) for 8 weeks after induction of NAFLD. The obtained results showed a significant increase in serum total cholesterol (TC) and triacylglycerols (TAG) concentrations with significant up-regulation of liver gene expression TNF- $\alpha$, IL- $1 \beta$, NF-k $\beta$, PPAR- $\alpha$, ACC, FAS and SREBT-1 in NAFLD- induced rats. Also, various histopathological alterations were detected in liver tissue of induced NAFLD rats. With spirulina treatment in NAFLD-induced rats, parameters measured near the normal level of rats group (G1). Also, spirulina potentially improved molecular hepatic function alterations related to NAFLD. Interestingly, histopathological findings supported that spirulina markedly attenuates harmful effects of NAFLD and protects liver tissue. We conclude that, spirulina has a great role as antioxidant, anti-obesity and anti-inflammatory via inhibition of liver lipid metabolic disturbance, steatosis and oxidative stress singling.
\end{abstract}

\section{INTRODUCTION}

Nonalcoholic fatty liver disease (NAFLD) is described by excessive fat accumulation in the liver. It ranges from steatosis to Nonalcoholic steatohepatitis (NASH). NAFLD is the most worldwide chronic liver diseases. It may followed by non-alcoholic steatosis, NASH, advanced fibrosis/cirrhosis, and Hepatocellular carcinoma (HCC) (Ahmed et al., 2019).

Obesity is characterized by abnormal or excessive fat accumulation that impairs health. Yang et al., (2020) evaluated and compared effects of Spirulina on obesity and hepatic lipid accumulation in high-fat and highsucrosediet induced obese mice. The accumulation of lipids in the liver results from an imbalance among hepatic lipid intake, synthesis, degradation and secretion. (Tessari et al., 2009)

Spirulina (Arthrospira platensis), a microscopic multicellular filamentous blue-green algae (cyano-bacterium), is rich in essential amino acids, polyunsaturated fatty acids (PUFAs), minerals, vitamins and carotenoids such as $\beta$ carotene and zeaxanthin. Recent researches talk about
Spirulina health advantagesas antioxidant, antiinflammatory, anti-cancer, anti-viral, anti-bacterial activities and protective effects against hyperlipidemia, malnutrition, obesity, diabetes (Hoseini et al., 2013).

Accordingly, this study aim to evaluate the possible protective effect of spirulina platensis against NAFLD induced by high fat diet in adult white male albino rats through investigation of serum lipids, oxidative stress and steatosis markers and inflammatory mediators of certain gene expression as well as histopathological examination of liver tissue.

\section{MATERIAL AND METHODS}

\subsection{Experimental animals:}

Twenty-one male albino rats, 8-10 weeks old and average body weight $160 \pm 10 \mathrm{~g}$ was used in this study. Rats were fed on constant ration; clean drinking water was supplied ad-libitum. All rats were acclimatized for 10 days prior to the beginning of study. Animal Care and Use committee at Benha University were approved this experimental protocols and were in accordance with the National

* Corresponding author emmm2006@yahoo.com 
Institute of Health Guide for the Care and Laboratory Animals.

\subsection{Chemicals and antioxidant agent: \\ 2.2.1-Spirulina platensis:}

Spirulina platensis was obtained in the form of powder from (National Research Centre in Giza, Egypt). Spirulina solution was prepared by dissolving 800 gram of spirulina powder in $20 \mathrm{ml}$ distilled water (Sixabela et al., 2011).

2.2.2-Propylene glycol, formaldehyde solution and normal saline:

Propylene glycol, formaldehyde solution and normal saline were purchased from ADWIC, El Nasr Pharmaceuticals Company for Trading Chemicals and Medical Appliances, Egypt

2.3. Experimental design:

Rats were acclimatizing to the laboratory conditions and partitioned into three equal groups randomly:

Group I: (Normal control group): Consisted of 7 rats, received no treatment, provided only with a constant standard pellet diet and plenty of clean water ad-libtium for 16 weeks.

Group II: (High fat diet-induced NAFLD group):Contained 7 rats received no treatment, and were fed on high fat diet ( $60 \%$ fat, $22 \%$ carbohydrates, $18 \%$ protein) daily for 8 weeks for induction of NAFLD, followed by ordinary basal ration feeding for another 8 weeks (Li et al., 2014).

Group III: (NAFLD+ Spirulina treated group): Included 7 rats fed HFD for 8 weeks for induction of (NAFLD) by the end of the $8^{\text {th }}$ week followed by basal ration feeding and treated with spirulina platensis orally $(150 \mathrm{mg} / \mathrm{kg}$ b.wt/day) for another 8 weeks (Sixabela et al., 2011).

\subsection{Sampling:}

2.4.1. Blood samples:

Blood samples were collected via veins puncture of the medial canthus of the eye, kept to clot, then centrifuge for 15 minutes at 3,000 rpm. Serum were separated in dry sterile tubes by automatic pipette, and then stored at -20 ${ }^{\circ} \mathrm{C}$ in a freezer until use for subsequent biochemical analysis.

\subsubsection{Liver for molecular analysis:}

All rats were sacrificed, after 16 week of the experiment, by cervical decapitation, the abdomen was open and the liver was rapidly excised gently, cleaned by rinsing with isotonic saline (ice-cold sodium chloride solution $0.9 \%$ ), immediately kept in liquid nitrogen and stored at $-80^{\circ} \mathrm{C}$ till RNA extraction for the real time quantitative polymerase chain reaction (qPCR) analysis of the hepatic gene expressions levels of Tumor necrosis factor- $\alpha$ (TNF- $\alpha$ ), Interleukin-1 $\beta$ (IL-1 $\beta)$, Nuclear factor- $\kappa \mathrm{B}$ (NF- $\mathrm{B} B$ ), Peroxisome proliferator-activated receptor- $\alpha$ (PPAR- $\alpha$ ), Acetyl-CoA carboxylase (ACC), Fatty acid synthase (FAS) and Sterol regulatory element binding protein-1c (SREBP-1).

\subsubsection{Liver for histopathological examination:}

Liver of rats in all groups were examined by naked eyes for any lesions after 16 weeks. Small liver specimen were taken and immediately fixed in natural buffered formalin $10 \%$ and tissue paraffin sections were routinely prepared and stain with $\mathrm{H}$ and $\mathrm{E}$ according to Bancroft and Gamble, (2008).

\subsection{Analysis:}

2.5.1. Biochemical analysis:

Serum triacylglycerols and total cholesterol concentrations were determined by enzymatic method described by Fossati and Prencipe (1982) and Allain et al., (1974), respectively.

2.5.2. Molecular analysis:

Real-time PCR with SYBR Green was used to measure expression of mRNAs of target genes in the liver. The isolated cDNA were amplified using 2X Maxima SYBR Green/ROX qPCR Master Mix following the manufacturer protocol and gene specific primers. The primers used in the amplification are shown in Table $\boldsymbol{A}$. The web based tool, Primer 3 (http://wwwgenome.wi.mit.edu/cgi-bin/primer/primer3_www.cgi) was used to design these primers based on published rat sequences.

Table A. Forward and reverse primers sequence for real time PCR.

\begin{tabular}{ccc}
\hline Gene & Forward primer(/5-----/3) & Reverse primer(/5 ------/3) \\
\hline TNF- $\alpha$ & GCATGATCCGCGACGTGGAA & AGATCCATGCCGTGGCCAG \\
IL-1 $\beta$ & CACCTCTCAAGCAGAGCACAG & GGGTCCATGGTGAAGTCAAC \\
NF-KB & CCTAGCTTCTCTGAACTGCAAA & GGGTCAGAGGCCAATAGAGA \\
PPAR- $\alpha$ & CCTGTCCGTCGGGATGTCACAC & GTGACGGTCTCCACGGACATGC \\
ACC & TGAGGAGGACCGCATTTATC & GCATGGAATGGCAGTAAGGT \\
FAS & GGATGTCAACAAGCCCAAGT & CAGAGGAGAAGGCACAAAG \\
SREBP-1 & GGCCCTGTGTGTACTGGTCT & AGCATCAGAGGGAGTAGGA \\
B-actin & AAGTCCCTCACCCTCCCAAAAG & AAGCAATGCTGTCACCTTCCC \\
\hline
\end{tabular}

\subsection{Statistical analysis:}

The statistical significance was evaluated by one-way analysis of variance (ANOVA) using SPSS, version 18.0 software (2011). All the data were expressed as means \pm S.E. The individual comparisons were obtained by Duncan's multiple range test (DMRT). Values were considered statistically significant when $(\mathrm{P}<0.05)$.

\section{RESULTS}

The obtained data illustrated in table (1) exhibited that serum total cholesterol and triacylglycerols concentrations were significantly increase $(\mathrm{P} \geq 0.01)$ in NAFLD group (G2) contrasted with control group (G1). Treatment of NAFLD with spirulina (G3) displayed significant decrease in serum total cholesterol and triacylglycerols concentrations $(\mathrm{P} \leq 0.01)$ contrasted with NAFLD group (G2).

Table 1 Effect of treatment with Spirulina platensis on serum total cholesterol and triacylglycerols concentrations in NAFLD-induced rats.

\begin{tabular}{lcc}
\hline \multicolumn{1}{c}{ Animal groups } & $\begin{array}{c}\text { Total } \\
\text { Cholesterol (mg/d) }\end{array}$ & $\begin{array}{l}\text { Triacylglycerol } \\
\mathrm{s}(\mathrm{mg} / \mathrm{dl})\end{array}$ \\
\hline Normal control (G1) & $88.69^{\mathrm{d}} \pm 4.45$ & $43.38^{\mathrm{d}} \pm 2.34$ \\
NAFLD (G2) & $177.25^{\mathrm{a}} \pm 8.92$ & $99.42^{\mathrm{a}} \pm 5.72$ \\
$\begin{array}{l}\text { NAFLD treated with Spirulina } \\
\text { (G3) }\end{array}$ & $131.29^{\mathrm{b}} \pm 6.68$ & $66.11^{\mathrm{b}} \pm 3.80$ \\
\hline
\end{tabular}

Data are presented as Mean \pm SEM (Standard error of mean)

Data are presented as Mean \pm SEM (Standard error of mean).
Mean values with different superscript letters in the same column are significantly different at $(\mathrm{P} \leq 0.05)$.

The current results (Table 2) uncovered a significant upregulation $(\mathrm{P} \leq 0.05)$ of $\mathrm{TNF}-\alpha$, Interleukin- $1 \beta$ and $\mathrm{NF}-\mathrm{k} \beta$ gene expression levels in liver tissue of NAFLD rats (G2) contrasted with normal control group (G1). This raised expression was significantly down regulated following administration of spirulina (G3). However, the expression levels of treated group (G3) remained significantly higher than the control group $(\mathrm{G} 1)$. 
Table 2 Effects of Spirulina platensis treatment on the relative expression of TNF- $\alpha$ and Interleukin-1 $\beta$ gene in liver tissues of NAFLD-induced rats.

\begin{tabular}{lcc}
\hline \multicolumn{1}{c}{ Animal groups } & \multicolumn{2}{c}{ Fold change (Relative } \\
& \multicolumn{2}{c}{ quantification)Mean \pm SEM } \\
& TNF- $\alpha$ & Interleukin-1 $\beta$ \\
\hline Normal control (G1) & $1.00^{\mathrm{e}} \pm 0.08$ & $1.00^{\mathrm{d}} \pm 0.08$ \\
NAFLD(G2) & $8.28^{\mathrm{a}} \pm 0.42$ & $6.45^{\mathrm{a}} \pm 0.29$ \\
NAFLD treated with spirulina (G3) & $4.47^{\mathrm{b}} \pm 0.20$ & $3.27^{\mathrm{b}} \pm 0.14$
\end{tabular}

Means within the same column carrying different superscript letters are significantly different $(\mathrm{P} \leq 0.05)$.

The obtained results presented in table (3) revealed a significant up regulation $(\mathrm{P} \leq 0.05)$ of PPAR- $\alpha$ and SREBT-1 gene expression level in liver of NAFLD rats (G2) as compared with control group (G1). However, the expression levels of the PPAR- $\alpha$ in spirulina treated group (G3) remained significantly higher and SREBT-1 gene expression was downregulated than the NAFLD rats $(\mathrm{G} 2)$. Table 3 Effects of Spirulinaplatensis treatment on the relative expression of NF-k $\beta$, PPAR- $\alpha$ and SREBT-1 gene in liver tissues of NAFLDinduced rats.

\begin{tabular}{|c|c|c|c|}
\hline \multirow[t]{3}{*}{ Animal groups } & \multirow{2}{*}{\multicolumn{3}{|c|}{$\begin{array}{l}\text { Fold change (Relative quantification) Mean } \pm \\
\text { SEM }\end{array}$}} \\
\hline & & & \\
\hline & NF-k $\beta$ & PPAR- $\alpha$ & SREBT-1 \\
\hline Normal control (G1) & $1.00^{\mathrm{d}} \pm 0.07$ & $1.00^{\mathrm{e}} \pm 0.08$ & $1.00^{\mathrm{d}} \pm 0.08$ \\
\hline NAFLD(G2) & $5.70^{\mathrm{a}} \pm 0.35$ & $2.75^{\mathrm{d}} \pm 0.16$ & $3.10^{\mathrm{a}} \pm 0.2$ \\
\hline NAFLD treated with spirulina & $3.12^{\mathrm{b}} \pm 0.16$ & $3.89^{\mathfrak{c}} \pm 0.2$ & $2.23^{\mathrm{b}} \pm 0.16$ \\
\hline
\end{tabular}

Means whe same column carrying different superscript letters are significantly different $(\mathrm{P} \leq 0.05)$

The obtained results (Table 4) revealed a significant up regulation $(\mathrm{P} \leq 0.05)$ of $\mathrm{ACC}$ and $\mathrm{FAS}$ gene expression level in liver of NAFLD rats (G2) contrasted with control group (G1). However, the expression levels of the spirulina treated group (G3) were significantly down regulated in comparison with the NAFLD rats (G2).

Table 4 Effects of Spirulina platensis treatment on the relative expression of ACC and FAS gene in liver tissues of NAFLD-induced rats.

\begin{tabular}{lcc}
\multicolumn{1}{c}{ Animal groups } & \multicolumn{2}{c}{ Fold change (Relative quantification) } \\
& ACC Mean \pm SEM & FAS \\
\hline Normal control (G1) & $1.00^{\mathrm{c}} \pm 0.08$ & $1.00^{\mathrm{e}} \pm 0.07$ \\
NAFLD(G2) & $4.08^{\mathrm{a}} \pm 0.2$ & $9.65^{\mathrm{a}} \pm 0.53$ \\
& & \\
NAFLD treated with spirulina (G3) & $2.69^{\mathrm{b}} \pm 0.16$ & $5.31^{\mathrm{b}} \pm 0.27$
\end{tabular}

Means within the same column carrying different superscript letters are significantly different $(\mathrm{P} \leq 0.05)$.

\section{Histopathological examination:}

Group I: (normal control group fed on normal ration):

The microscopic examination of the liver revealed hepatocytes arranged in trabeculae running radially from the central vein and separated by sinusoids (Fig. 1).

GroupII: (Nonalcoholic fatty liver disease group):

The microscopical examination of liver showed marked degree of hepatic steatosis, severe congestion of central vein and portal blood vessels. Moreover, the hepatic tissue suffers from severe degree of degenerative changes in the form of hydropic degeneration. Also focal area of necrosis was also detected among hepatic parenchyma which presented by pyknotic nuclei with more esinophilic hepatic cytoplasm (Figs.2 and 3).

Group III: (Non alcoholic fatty liver disease treated with Spirulina):

Microscopically the examined liver showed improvement in the microscopic picture of the examined liver where mild degenerative hepatic changes were only the detectable lesion (Fig. 4)

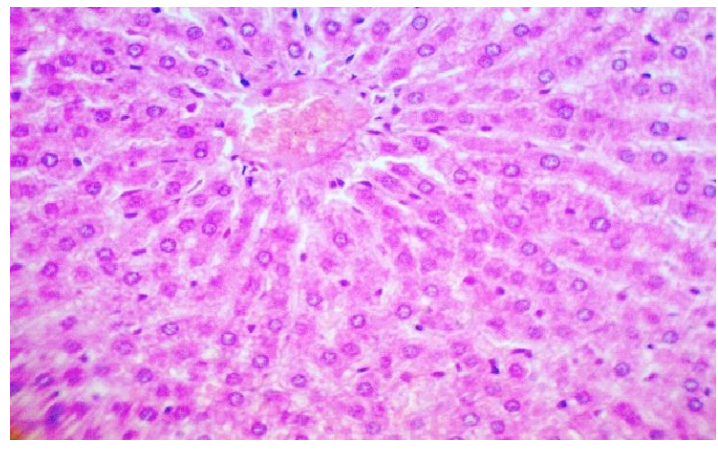

Figure (1): Hematoxylin and eosin stained liver section of a rat from control group showing hepatocytes arranged in trabeculae running radially from the central vein and separated by sinusoids. $(\times 200)$.

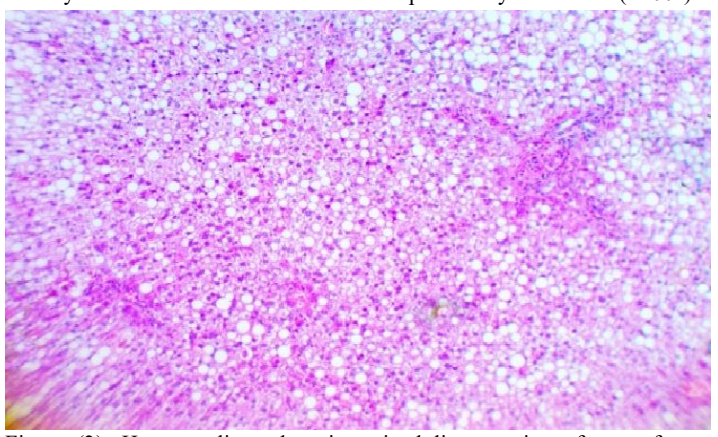

Figure (2): Hematoxylin and eosin stained liver section of a rat from NAFLD group, showing marked degree of hepatic steatosis with multiple necro-inflammatory foci in hepatic parenchyma $(\times 100)$.

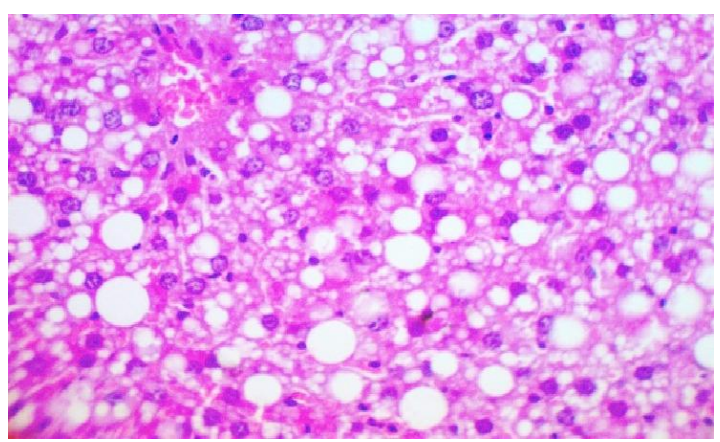

Figure (3): Hematoxylin and eosin stained liver section of a rat from NAFLD group, showing marked degree of hepatic steatosis $(\times 200)$.

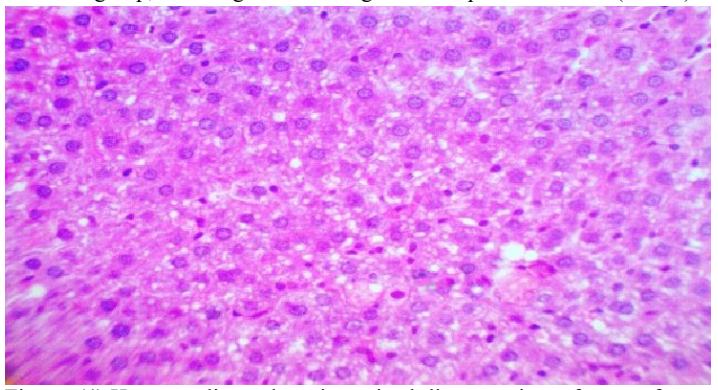

Figure (4):Hematoxylin and eosin stained liver section of a rat from NAFLD treated with spirulina group at a dose of $(150 \mathrm{mg} / \mathrm{kg} /$ day $)$, showing vacuolation of some hepatocytes $(\times 200)$.

\section{DISCUSSION}

The results of this investigation revealed a significant increase in serum total cholesterol and triacylglycerols concentrations in high-fat diet-induced NAFLD group (G2) when compared with control group (G1). This in agreement with Miller et al. (2011) who expressed that serum triacylglycerols, total cholesterol (TC), and Low- 
density lipoprotein cholesterol (LDL-c) levels were significantly increased after utilization of high fat eating routine while levels of HDL-c reduced at the same time. However, treatment of NAFLD with spirulina (G3) exhibited significant decrease in serum total cholesterol and triacylglycerols concentrations when compared with NAFLD group (G2). Similarly, (Zhao et al., 2019) revealed that Blue-green algae known as (Spirulina platensis) have anti-obesity effects. It diminishes body weight, reduces serum glucose level and diminishes total cholesterol concentration.

A significant $(\mathrm{P} \leq 0.05)$ up regulation of the relative expression of TNF- $\alpha$ and IL- $1 \beta$ gene were observed in liver tissues of NAFLD-induced rats (G2) when contrasted with normal control group (G1). Meanwhile, treatment of NAFLD with spirulina (G3) exhibited significant down regulation of liver TNF- $\alpha$ and IL-1ßgene expression levels when compared with NAFLD group (G2).These results are in accordance with Meeks et al., (2000) who reported that inflammatory markers TNF- $\alpha$, IL-- $1 \beta$ levels were expanded and this indicates the activation of macrophages. Also, Sagara et al., (2015) express that antioxidant and free radical-scavenging properties of Spirulina platensis may be because of its phycocyanin content. Likewise, Sin et al. (2014) and Ali et al. (2015), establish that Spirulina inhibited expression of genes encoding TNF- $\alpha$.

The existing results revealed a significant $(\mathrm{P} \leq 0.05)$ upregulation of ACC, SREBT-1 and FAS gene expression in liver of NAFLD- induced in rats (G2) when compared with control group (G1). This elevated expression was significantly down regulated following administration of spirulina (G3). This data are in agreement with Hirahashi et al. (2002) who demonstrated that spirulina treatments significantly reduce pro-inflammatory cytokines and this confirms the anti-inflammatory characteristic of spirulina. Moreover, Grahame (2014) demonstrated anti-obesity effects of spirulina as spirulina treatment regulates hepatic architecture and significantly diminishes lipid droplets. And downstream genes, such as SREBP-1c and ACC regulated the lipid metabolism problems, in accordance with the presented results which explained that ACC gene expression decreased in spirulina treated group (G3) when contrasted with NAFLD group (G2). Furthermore, Yoon, (2009) suggest that spirulina diminished hepatic lipid via managing the pathway of SREBP-1c-mediated gene transcription. It has been shown that PPAR- $\alpha$ activators can control obesity in rats through down circulating triacylglycerols (TAG) and increasing the hepatic fatty acid oxidation, this are nearly similar to the obtained results that revealed the expression levels of the PPAR- $\alpha$ in spirulina treated group (G3) remained significantly higher and SREBT-1 gene expression was down regulated than the NAFLD rats (G2). This suggestion was supported with Yang et al., 2020 who showed that dietary Spirulina up-regulated the hepatic mRNA levels of PPAR $\alpha$, CPT$1 \alpha$ in high fat eating routine mice and this was associated with the enhancement in the mRNA expression of PPAR$\alpha$, this results are in accordance with the existing data that shown enhancement of PPAR- $\alpha$ gene expression in spirulina treatment of NAFLD.

\section{CONCLUSION}

It could be concluded that, Spirulina treatment has a hepatoprotective and strong anti-inflammatory effect against NAFLD by enhancing antioxidant defense system, and has a strong anti-inflammatory effect through modulation of SREBP- $1 \mathrm{c}, \mathrm{NF}-\kappa \mathrm{B}$, and PPAR- $\alpha$ gene expressions, and anti-obesity by increasing the hepatic fatty acid oxidation and regulate fat metabolism.

\section{REFERENCES}

1. Ahmed, I., Mikail, M., Mustafa, M., Ibrahim, M., Othman, R. 2019. Lifestyle interventions for non-alcoholic fatty liver disease. Saudi Journal of Biological Sciences 26,1519-1524.

2. Ali, E., Barakat, B., Hassan, R. 2015. Antioxidant and angiostatic effect of Spirulinaplatensis suspension in complete Freund's adjuvant-induced arthritis in rats. PLoS ONE 10(4), e0121523.

3. Allain, C., Poon, L., Chan, C., Richmond, W., Fu, P. 1974. Enzymatic Determination of Total Serum Cholesterol. Clinical Chemistry 20(4), 470-475.

4. Bancroft, J.D., Gamble, M. 2008. Theory and Practice of Histological Techniques. $6^{\text {th }}$ ed. Churchill Livingstone, Elsevier.

5. Fossati, P., Prencipe L. 1982. Serum triglycerides determined colorimetrically with an enzyme that produces hydrogen peroxide. Clinical Chemistry 28(10), 2077-2080.

6. Grahame, H. 2014. AMP-activated protein kinase: a key regulator of energy balance with many roles in human disease. J Intern Med 276, 543-559.

7. Hirahashi, T., Matsumoto, M., Hazeki, K., Saeki, Y., Ui, M., Seya, T. 2002. Activation of the human innate immune system by Spirulina: augmentation of interferon production and NK cytotoxicity by oral administration of hot water extract of Spirulina platensis. Int Immunopharmacol 2(4), 423-434.

8. Hoseini, S., Khosravi-Darani, K., Mozafari, M. 2013. Nutritional and medical applications of spirulina microalgae. Mini Rev Med Chem 13(8), 1231-1237.

9. Li, S., Meng, F., Liao, X., Wang, Y., Sun, Z., Guo, F., Li, X., Meng, M., Li, Y., Sun, C. 2014. Therapeutic Role of Ursolic Acid on Ameliorating Hepatic Steatosis and Improving Metabolic Disorders in High-Fat Diet-Induced Non-Alcoholic Fatty Liver Disease Rats. PLoS ONE 9(1), e86724.

10. Meeks, R., Harrison, S., Bull, R. 2000. Hepatotoxicity, biochemical methods of studying hepatotoxicity chapter. In: Raos, Prasada, Kodacanti, Hehendale, Harhara M., (Eds.), CRC Press Inc., Florida, USA, p. 267.

11. Miller, M., Stone, N., Ballantyne, C., Bittner, V., Criqui, M., Ginsberg, H., Goldberg, A., Howard, W., Jacobson M., Kris-Etherton, P. 2011. Triglycerides and cardiovascular disease: a scientific statement from the American Heart Association Circulation 123, 2292-2333.

12. Sagara, T., Nishibori, N., Kishibuchi, R. 2015. Non-protein components of Arthrospira (Spirulina) platensis protect PC12 cells against iron-evoked neurotoxic injury. J ApplPhycol 27(2), 849-855.

13. Sin, J., Choi, W., Lee, H. 2014. Comparison of antiinflammatory activity of Spirulina maxima extract by ultrasonication and water extraction process Spirulina maxima. J Korean Soc Food Nutr 43(12), 1852-1857.

14. Sixabela, P., Chivandi E., Badenhorst M. and Erlwanger K. (2011): The Effects of Dietary Supplementation with Spirulina platensis in Growing Rats. Asian Journal of Animal and Veterinary Advances 6, 609-617.

15. Tessari, P., Coracina, A., Cosma, A., Tiengo, A. 2009. Hepatic lipid metabolism and non-alcoholic fatty liver disease. Nutrition, metabolism, and cardiovascular diseases: NMCD 19(4), 291-302

16. Yang, Y., Duc, L., Hosokawaa, M., Miyashitaa, K. 2020. Effect of Spirulina lipids on high-fat and high-sucrose diet induced obesity and hepatic lipid accumulation in C57BL/6J mice. Journal of Functional Foods 65, 103741.

17. Yoon, M. 2009. The role of PPAR $\alpha$ in lipid metabolism and obesity: Focusing on the effects of estrogen on PPAR $\alpha$ action. Pharmacological Research 60 (3) 151-159.

18. Zhao, B., Cui, Y., Fan, X., Qi, P., Liu, C., Zhou, X., Zhang, X. 2019. Anti-obesity effects of Spirulinaplatensis protein hydrolysate by modulating brain-liver axis in high-fat diet fed mice. PLoS One 14(6), e0218543. 